\title{
Salivary cytokines as biomarkers of oral cancer: a systematic review and meta- analysis
}

Mayara Martina Abatti Chiamulera 1,2,3, Caroline Biazzolo Zancan ${ }^{1,2}$, Aline Pertile Remor ${ }^{1,2}$, Marcos Freitas Cordeiro ${ }^{1,2}$, Frederico Omar Gleber-Netto ${ }^{4}$ and Antuani Rafael Baptistella ${ }^{1,2,3^{*}}$ (D)

\begin{abstract}
Background: Oral cancer (OC) is usually diagnosed at advanced clinical stages due to its asymptomatic nature and absence of pathognomonic signs in its early development phase. Delayed diagnosis is one of the major causes of OC treatment failure and poor prognosis. Development of alternative diagnostic approaches are imperative for improving early detection and therapeutic success rates. Salivary cytokines (SC) have been studied as potential diagnostic biomarkers for $\mathrm{OC}$ and may represent a potential tool for improvement of its early detection.

Methods: In this systematic review and meta-analysis we identified SC studied as OC biomarkers by systematically reviewing the PubMed and Cochrane Library databases using the terms: "oral cancer", "cytokine", and "saliva", and also combined with "interleukin" or "interferon". Only case-control studies that measured SC by ELISA from treatment nairve patients were included in the qualitative review. For the meta-analysis were included all comparable studies that provided enough data (sample size, mean and standard deviation or standard error of the mean) for SC levels in OC patients, non-cancer controls and patients with oral potentially malignant disorders (OPMD), including leukoplakia. Comparisons with patients with oral lichen planus (OLP) and gingivitis were included in the qualitative analysis.
\end{abstract}

Results: A total of 28 articles (from 2004 to 2018) were included in the systematic review, describing 10 different SC, being IL-8 and IL- 6 the most studied ones. SC levels were consistently higher among OC patients when compared to healthy controls and to patients with OPMD, OLP and gingivitis. Meta-analysis including 23 eligible studies showed that IL-8, IL-6, TNF-a, IL-1 $\beta$ and IL-10 salivary levels were significantly higher in OC patients compared to controls; and that IL-8, IL-6, TNF- $\alpha$ and IL-1 $\beta$ salivary levels were also higher in OC patients compared to individuals with OPMD. When compared to healthy controls, OPMD patients showed significantly higher IL-6 and TNF-a salivary levels.

\footnotetext{
* Correspondence: antuani.baptistella@unosec.edu.br

'Universidade do Oeste de Santa Catarina (UNOESC), Joacaba, SC, Brazil

${ }^{2}$ Programa de Pos-Graduacao em Biociencias e Saude/Universidade do Oeste de Santa Catarina, Joacaba, SC, Brazil

Full list of author information is available at the end of the article
}

(c) The Author(s). 2021 Open Access This article is licensed under a Creative Commons Attribution 4.0 International License, which permits use, sharing, adaptation, distribution and reproduction in any medium or format, as long as you give appropriate credit to the original author(s) and the source, provide a link to the Creative Commons licence, and indicate if changes were made. The images or other third party material in this article are included in the article's Creative Commons licence, unless indicated otherwise in a credit line to the material. If material is not included in the article's Creative Commons licence and your intended use is not permitted by statutory regulation or exceeds the permitted use, you will need to obtain permission directly from the copyright holder. To view a copy of this licence, visit http://creativecommons.org/licenses/by/4.0/ The Creative Commons Public Domain Dedication waiver (http://creativecommons.org/publicdomain/zero/1.0/) applies to the data made available in this article, unless otherwise stated in a credit line to the data. 


\begin{abstract}
(Continued from previous page)
Conclusions: Our analyses showed that the salivary levels of some cytokines are consistently different among OC, OPMD and healthy patients, indicating that these SC may represent potential diagnostic biomarkers for OC and OPMD. Despite of that, SC levels were highly variable among studies, suggesting that further technical improvement and standardization for SC measurement by ELISA is needed in order to successfully translate these biomarkers to the clinical practice.
\end{abstract}

Keywords: Oral cancer, Head and neck cancer, Saliva, Cytokines, Biomarkers

\section{Background}

Oral squamous cell carcinoma (OC) is the most common type of oral cancer, and represents $\sim 2 \%$ of all cancer cases in the USA. More than $50 \%$ of OC patients are diagnosed at advanced clinical stage, with large primary tumors and nodal or distant dissemination. Patients with advanced OC are usually treated with multimodal therapeutic approaches, which are poorly effective and linked to high morbidity $[1,2]$. The 5 -year survival rate for advanced OC patients is around 50\%, while $85 \%$ survival rate is observed for early-stage patients [3, 4]. Thus, early diagnosis is a major prognostic factor for OC patients.

Unfortunately, diagnosis of $\mathrm{OC}$ at early stages is challenging. Detection of early lesions is usually incidental, since they are usually asymptomatic and rarely perceived by patients. Clinically, early OC lesions may resemble other benign oral mucosal conditions, which can lead to delayed diagnosis. Also, many of the $\mathrm{OC}$ cases are preceded by lesions with considerable potential for malignant transformation (mostly oral leukoplakia), which are collectively named as oral potentially malignant disorders (OPMD). Although only $2 \%$ of the OPMD will eventually turn into cancer, determination of its malignant transformation risk and distinction from early stage OC are quite challenging [4-7]. Thus, histopathological assessment is imperative for definitive diagnosis which can contribute to further delay in OC detection $[8,9]$.

Considering that, many research groups have been seeking for early OC detection biomarkers, and differentiate it from benign lesions with similar clinical features. Because saliva is in intimate contact with the oral mucosa, it has been widely studied as a source of OC biomarkers [10]. Saliva carries molecules and cells originated in the aero-digestive tract, as well as nucleic acids and proteins that are passively and actively transported from the circulatory system into the salivary glands [11]. In this way, it has been suggested that saliva has a similar potential as a source of biomarkers as the blood, but with the advantage of being obtained by noninvasive, inexpensive, and safer techniques [12].

Several exploratory case-control studies found that salivary cytokines $(\mathrm{SC})$ were highly deregulated in $\mathrm{OC}$ patients [13-16]. Considering the ubiquitous role of cytokines in human diseases [17], subsequent studies investigated whether SC levels would be different between OC patients and individuals with inflammatory or benign oral mucosa conditions [18-20]. These studies supported the association between SC and OC, corroborating with the hypothesis that SC are potential OC diagnosis biomarkers.

Despite of that, there is still no consensus on the discriminatory power for each SC, or whether these cytokines are able to differentiate OC from OPMD and other inflammatory oral diseases. Therefore, the objective of this systematic review and meta-analysis is to identify the $\mathrm{SC}$ with potential diagnostic power for $\mathrm{OC}$ detection and to verify whether the levels of these SC are consistent among different studies. The results presented here are of great relevance for guiding future technical and clinical endeavors seeking the development of early stage OC biomarkers based on SC, which is an essential step towards treatment improvement for OC patients.

\section{Methods}

This review was performed following the Cochrane Handbook for Systematic Reviews of Interventions (Version 5.1.0) [21], also registered and published at the International prospective register of systematic reviews (PROSPERO - $\mathrm{n}^{\circ}$ CRD42018111397). Data search, screening and extraction were executed by two of the authors (MMAC and CBZ).

Data search was performed using the electronic databases PubMed and Cochrane Library, considering manuscripts published between 1950 and 2019 and 1999 and 2019, respectively. The searching terms used were: "cytokine", "oral cancer" and "saliva", and also combined with "interleukin" or "interferon". The searching period was from September 3rd, 2018 to January 31st, 2019.

The resulting manuscripts were initially screened by title and abstract, followed by a full-text analysis. Duplicates were checked manually and removed by two of the authors (MMAC and CBZ). Manuscripts were then selected based on the following inclusion criteria:

- Publication type: Peer-reviewed original articles, published in the English language; 
- Study design: Case-control studies with human subjects

- Exposure of interest: Participants enrolled in the "case" group diagnosed with OC and "controls" corresponding to healthy subjects. For both groups, SC should have been measured in its protein form.

- Method of sampling: SC measured using enzymelinked immunosorbent assay (ELISA).

- Research question: Studies comparing SC levels between OC patients, non-cancer control subjects and patients with OPMD or leukoplakia.

In case of discordance about the eligibility criteria, a third author (ARB) was consulted.

\section{Data extraction}

A database was created to organize the information from the selected publications, including author details, year of publication, number of patients, and mean SC expression values with standard deviation or standard error of the mean. Two authors (MMAC and CBZ) analyzed the data and a third author (ARB) re-evaluated all the information.

The selected articles were further evaluated considering the following aspects: whether the period of sample collection was reported; if the study was prospective; if the techniques for saliva collection, storage, and analysis were standardized and properly reported.

\section{Data analysis}

The data used for the meta-analysis were sample size, mean and standard deviation of the expression of SC in healthy controls, OC patients and patients with OPMD, including leukoplakia. Studies lacking any of these information were excluded. All data for the standard errors of the mean (SEM) were converted to standard deviations (SD) and all measurements were converted to picograms of protein per milliliter of saliva $(\mathrm{pg} / \mathrm{mL})$, and studies were excluded when this conversion was not possible. Measurements that were observed in single studies were also excluded from the meta-analysis because of the impossibility to estimate a combined effect. All these findings, however, were included in the qualitative analysis.

The random effects meta-analysis was conducted using the "meta" package in R. The standard mean difference (SMD) was estimated using the Hedges' g, and the in-between study variance $\left(\tau^{2}\right)$ was calculated using the DerSimonian-Laird estimator. All in-text data is represented as SMD followed by $95 \%$ confidence intervals $(\mathrm{CI})$.

\section{Results}

The initial search in electronic databases PubMed (1950-2019) identified 182 studies. After screening, a total of 28 studies were included into our qualitative analysis (Fig. 1). Among the selected articles, PolzDacewicz et al. (2016) reported SC concentrations in nanograms of protein per milliliter of saliva $(\mathrm{ng} / \mathrm{mL})$, which were converted to picograms of protein per milliliter of saliva $(\mathrm{pg} / \mathrm{mL})$ aiming to standardize their findings with the other studies. Gonçalves et al. (2015) was not included in the meta-analysis because the measurements were reported in picograms of SC per milligram of total salivary protein $(\mathrm{pg} / \mathrm{mg})$. Four studies were excluded because of lacking information concerning SD or SEM.

The selected 28 studies were all published between 2004 and 2018, from 12 different countries and the sample size ranged from 18 to 300 patients. The control groups were composed by healthy individuals, patients with OPMD, oral lichen planus (OLP), or periodontitis (Tables 1, 2, 3, 4, 5 and 6). The total number of patients, combining the 28 studies, considering each SC evaluated and each study group are described in the Fig. 2. IL-8 was investigated in 1245 individuals, followed by IL- 6 and Tumor Necrosis Factor $\alpha$ (TNF $\alpha)$, investigated in 963 and 724 individuals, respectively. In total, the SC identified in the frequency of appearance in these studies were IL-8 (50\%), IL-6 (50\%), TNF- $\alpha$ (28.6\%), IL-1 $\beta$ (21.4\%), IL10 (17.9\%), IL-1 $\alpha$ (10.7\%) and IL-1, IL-1RA, IL-4 and IL-13 (3.6\% each) (Fig. 2a). Among the studied groups in the 28 selected studies, OC had the higher number of individuals included (1670), followed by 1574 healthy subjects, 667 patients with OPMD, 108 with OLP and 62 with periodontitis (Fig. 2b). The number of occurrences of each SC for each condition is summarized in Fig. 2c.

The meta-analysis comparing the concentration of SC in OC patients versus healthy controls showed a significant increase in the level of IL-8 (standardized mean difference $(\mathrm{SMD})=1.77 ; 95 \% \mathrm{CI} 0.79$ to 1.55$)$, IL-6 $(\mathrm{SMD}=2.08 ; 95 \%$ CI 1.33 to 2.84$)$, TNF- $\alpha(\mathrm{SMD}=2.04$; $95 \%$ CI 0.47 to 3.61 ), IL-1 $\beta$ (SMD $=0.78 ; 95 \%$ CI 0.44 to $1.13)$, and IL-10 (SMD $=0.46 ; 95 \%$ CI 0.05 to 0.86$)$ in the cancer group. IL- $1 \alpha$ was the only SC that did not present a significant difference $(\mathrm{SMD}=2.21 ; 95 \% \mathrm{CI}-$ 0.36 to 4.77 ) (Fig. 3). IL-1, IL-1RA, IL-4 and IL-13 were excluded from the analysis because there was just a single observation for each.

Most likely due to variations in experimental procedures for saliva collection and SC quantification, inbetween studies heterogeneity was high in most cases. When comparing OC vs. controls, heterogeneity was non-significant only for IL-10 ( $\left.\mathrm{I}^{2} 46 \%, p=0.16\right)$. 


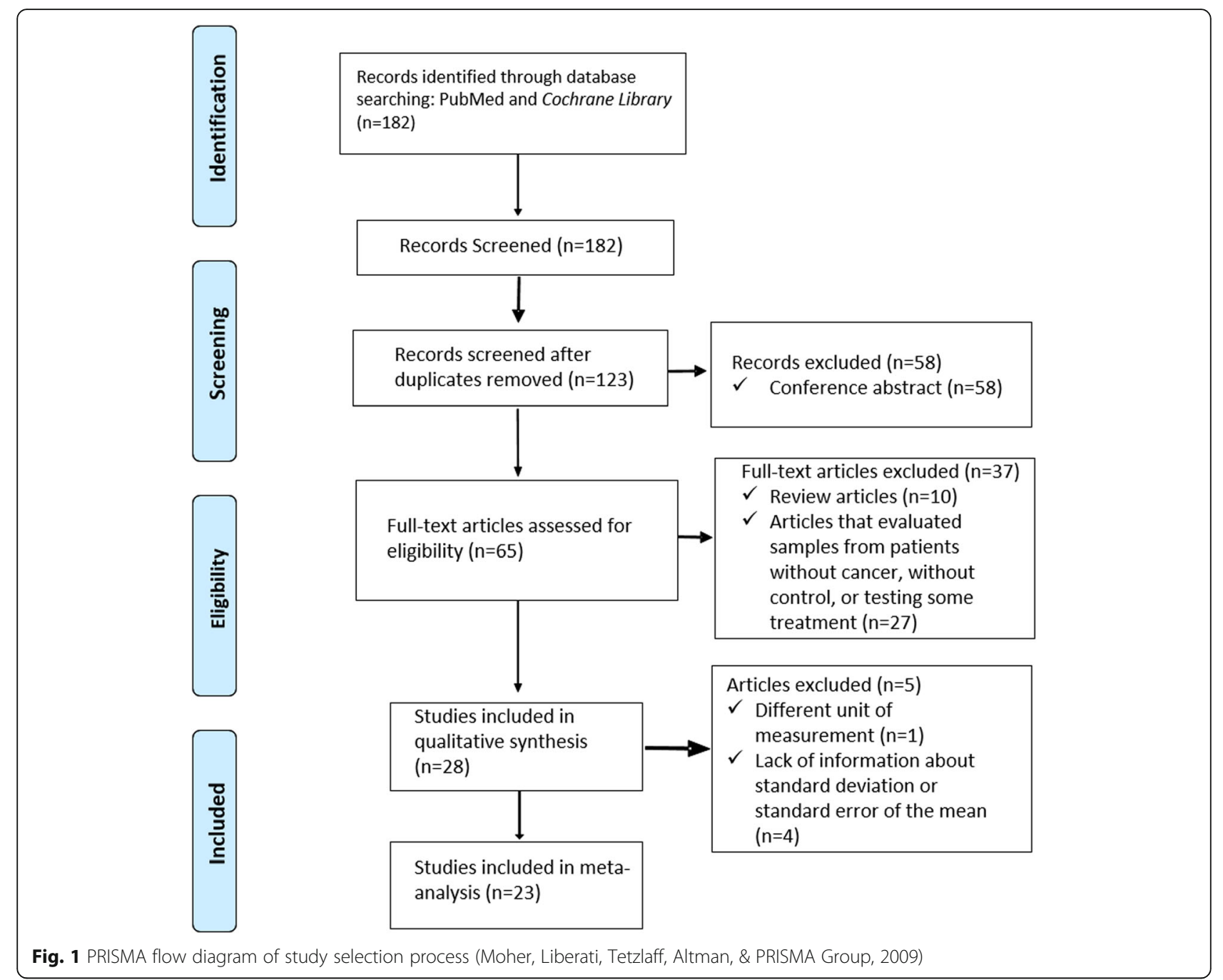

\section{Interleukin-8}

IL-8 levels in OC patients were compared to controls in 14 articles. The study with the smaller population of OC included five patients (Cheng, S. et al. 2014), and the largest one evaluated SC concentration in 100 OC patients (Rajkumar, K. et al. 2014). Among these 14 articles, 14 groups of OC, 5 groups of OPMD patients, 2 groups of OLP and 2 groups of periodontitis patients were evaluated. In all these studies, IL- 8 levels were reported to be significantly different between OC patients and controls. The values of IL-8 in control group varied from 52.1 to $1580.7 \mathrm{pg} / \mathrm{mL}$; for the premalignant group, values between 140.3 and $1918.2 \mathrm{pg} / \mathrm{mL}$ were found; and in the OC group, the values were between 283.7 and 4082.8 $\mathrm{pg} / \mathrm{mL}[14,22-34]$. Out of these 14 studies, 11 were eligible for meta-analysis, and the combined effect was significant, indicating an increase in the concentration of this $\mathrm{SC}$ in $\mathrm{OC}$ patients comparison to controls $\mathrm{SMD}=$ 1.77; $95 \%$ CI 0.79 to 1.55 ) (Fig. 3). However, when comparing IL-8 salivary concentration in OPMD patients against healthy controls, it was not significant with a bordering value $(\mathrm{SMD}=0.20 ; 95 \% \mathrm{CI} 0.00$ to 0.40 ), and heterogeneity was very low $\left(\mathrm{I}^{2}=0 \%, p=0.53\right)$ (Fig. 4a). IL-8 concentration in OC patients was significantly higher than what was observed in OPMD patients $(\mathrm{SMD}=0.97 ; 95 \%$ CI 1.81 to 0.13$)$, but heterogeneity was very high $\left(\mathrm{I}^{2}=92 \%, p<0.01\right)$ (Fig. $\left.4 \mathrm{~b}\right)$.

\section{Interleukin-6}

Interleukin-6 (IL-6) was evaluated in 14 articles, and in all of them the amount of IL- 6 was reported to be statistically higher in OC patients compared to the control group. The population of the studies varied from 9 subjects in the smallest study, to 100 subjects in the largest one. Five articles only compared OC patients with a control group of non-cancer people, while 07 articles also compared the OC with OPMD, and one article compared OC patients with a non-cancer group with periodontitis. The IL-6 values for healthy subjects varied from 0 to $16.0 \mathrm{pg} / \mathrm{mL}$ in ten of the articles $[18,20,22$, 
Table 1 Salivary interleukin 8 in oral cancer

\begin{tabular}{|c|c|c|c|c|c|c|c|c|}
\hline Cytokine & Author & Year & Groups & $\begin{array}{l}\mathrm{n} \text { of } \\
\text { cases }\end{array}$ & $\begin{array}{l}\text { Mean } \pm \\
\mathrm{SD}(\mathrm{pg} / \\
\mathrm{ml})\end{array}$ & Statistic test & $p$ Value & AUC \\
\hline \multirow[t]{22}{*}{ IL 8} & \multirow[t]{2}{*}{$\begin{array}{l}\text { SAHEBJAMEE M. } \\
\text { et al. }\end{array}$} & \multirow[t]{2}{*}{2008} & Oral Cancer & 9 & $\begin{array}{l}1093.7 \pm \\
1089.0\end{array}$ & \multirow[t]{2}{*}{$\begin{array}{l}\text { Kolmogorov-Smirnov Test } \\
\text { and Mann-Whitney U }\end{array}$} & \multirow[t]{2}{*}{$<0.05$} & \multirow[t]{2}{*}{-} \\
\hline & & & Control & 9 & $\begin{array}{l}700.7 \pm \\
1031.5\end{array}$ & & & \\
\hline & \multirow[t]{4}{*}{$\begin{array}{l}\text { ELASHOFF, D. } \\
\text { et al. }\end{array}$} & \multirow[t]{4}{*}{2012} & $\begin{array}{l}\text { Oral Cancer - } \\
\text { cohort } 4\end{array}$ & 36 & $\begin{array}{l}2563.0 \pm \\
2179.0\end{array}$ & \multirow[t]{4}{*}{$\begin{array}{l}\text { Mann-Whitney } U \text { test and } \\
\text { area under the curve (AUC) }\end{array}$} & \multirow[t]{2}{*}{$<0.05$} & \multirow[t]{4}{*}{0.680} \\
\hline & & & Control - cohort 4 & 54 & $\begin{array}{l}808.0 \pm \\
1132.0\end{array}$ & & & \\
\hline & & & $\begin{array}{l}\text { Oral Cancer - } \\
\text { cohort } 5\end{array}$ & 31 & $\begin{array}{l}2140.0 \pm \\
2282.0\end{array}$ & & $<0.05$ & \\
\hline & & & Control - cohort 5 & 70 & $\begin{array}{l}739.0 \pm \\
1002.0\end{array}$ & & & \\
\hline & \multirow{4}{*}{$\begin{array}{l}\text { ARELLANO- } \\
\text { GARCIA, M.E. } \\
\text { et al. }\end{array}$} & \multirow[t]{4}{*}{2008} & Oral Cancer & 40 & $\begin{array}{l}3347.7 \pm \\
2929.0\end{array}$ & \multirow{4}{*}{$\begin{array}{l}\text { Student t-test, Pearson } \\
\text { correlation coefficient; ROC } \\
\text { curve and area under curve }\end{array}$} & \multirow{4}{*}{$\begin{array}{l}\text { With } \\
\text { single-plex: } \\
0.02 \\
\text { With } \\
\text { multiplex: } \\
0.04\end{array}$} & \multirow{4}{*}{$\begin{array}{l}0.820 \\
(\text { Sensitivity }=87.5 \% \\
\text { Specificity }=64.3 \%)\end{array}$} \\
\hline & & & $\begin{array}{l}\text { Oral Cancer } \\
\text { Control }\end{array}$ & 42 & $\begin{array}{l}759.4 \pm \\
563.0\end{array}$ & & & \\
\hline & & & Periodontitis & 10 & $\begin{array}{l}818.8 \pm \\
228.4\end{array}$ & & & \\
\hline & & & $\begin{array}{l}\text { Periodontitis } \\
\text { Control }\end{array}$ & 10 & $\begin{array}{l}589.2 \pm \\
370.3\end{array}$ & & & \\
\hline & \multirow[t]{2}{*}{ TANN, W. et al. } & \multirow[t]{2}{*}{2007} & Oral cancer & 20 & $\begin{array}{l}1252.0 \pm \\
456.0\end{array}$ & \multirow[t]{2}{*}{ T-test and area under curve } & \multirow[t]{2}{*}{$<0.00001$} & \multirow[t]{2}{*}{0.837} \\
\hline & & & Control & 20 & $\begin{array}{l}577.0 \pm \\
355.0\end{array}$ & & & \\
\hline & \multirow{2}{*}{$\begin{array}{l}\text { KATAKURA A. } \\
\text { et al. }\end{array}$} & \multirow[t]{2}{*}{2007} & Oral Cancer & 19 & 720.0 & \multirow[t]{2}{*}{ Two-tailed t-tests } & \multirow[t]{2}{*}{$<0.05$} & \multirow[t]{2}{*}{-} \\
\hline & & & Control & 20 & 250.0 & & & \\
\hline & \multirow[t]{3}{*}{$\begin{array}{l}\text { RAJKUMAR, K. } \\
\text { et al. }\end{array}$} & \multirow[t]{3}{*}{2014} & Oral Cancer & 100 & $\begin{array}{l}1091.7 \pm \\
167.1\end{array}$ & \multirow{3}{*}{$\begin{array}{l}\text { Shapiro-Wilk's test, Kruskal- } \\
\text { Wallis analysis, Mann-Whitney } \\
\text { U test, Spearman rank test, } \\
\text { Receiver operator characteristic } \\
\text { (ROC) curve and area under } \\
\text { curve }\end{array}$} & \multirow[t]{3}{*}{$<0.05$} & \multirow{3}{*}{$\begin{array}{l}\mathrm{PML} \times \mathrm{OSCC}=0.971 \\
(95 \% \mathrm{Cl}=0.953-0.990 ; \\
p<0.0001)\end{array}$} \\
\hline & & & Premalignant & 100 & $\begin{array}{l}650.4 \pm \\
207.3\end{array}$ & & & \\
\hline & & & Control & 100 & $\begin{array}{l}349.6 \pm \\
115.1\end{array}$ & & & \\
\hline & $\begin{array}{l}\text { CHENG, L.Y.S. } \\
\text { et al. }\end{array}$ & 2014 & Oral Cancer & 5 & $\begin{array}{l}1525.3 \pm \\
1123.9\end{array}$ & $\begin{array}{l}\text { Kruskal-wallis test Mann- } \\
\text { Whitney U test (post hoc) }\end{array}$ & $<0.001$ & - \\
\hline & & & $\begin{array}{l}\text { Chronic } \\
\text { periodontitis }\end{array}$ & 21 & $\begin{array}{l}738.8 \pm \\
394.0\end{array}$ & & & \\
\hline & & & $\begin{array}{l}\text { Disease active - } \\
\text { Oral lichen planus }\end{array}$ & 15 & $\begin{array}{l}1328.4 \pm \\
731.8\end{array}$ & & & \\
\hline & & & $\begin{array}{l}\text { Disease inactive - } \\
\text { Oral lichen planus }\end{array}$ & 13 & $\begin{array}{l}1083.1 \pm \\
646.2\end{array}$ & & & \\
\hline & & & Control & 21 & $\begin{array}{l}890.8 \pm \\
563.2\end{array}$ & & & \\
\hline Cytokine & Author & Year & Groups & $\begin{array}{l}\text { Sample } \\
\text { (n) }\end{array}$ & $\begin{array}{l}\text { Mean } \pm \\
\text { SD (pg/ } \\
\mathrm{ml})\end{array}$ & Statistic test & P Value & AUC \\
\hline IL 8 & $\begin{array}{l}\text { PUNYANI, S.R.; } \\
\text { SATHAWANE, }\end{array}$ & 2013 & Oral Cancer & 25 & $\begin{array}{l}1718.6 \pm \\
668.3\end{array}$ & $\begin{array}{l}\text { Scheffe's analy- sis and } \\
\text { two-tailed independent }\end{array}$ & $p<0.0001$ & - \\
\hline & & & $\begin{array}{l}\text { Oral Precancer and } \\
\text { Oral leukoplakia }\end{array}$ & 25 & $\begin{array}{l}299.5 \pm \\
158.2\end{array}$ & sa & & \\
\hline & & & Control & 25 & $\begin{array}{l}210.1 \pm \\
142.3\end{array}$ & & & \\
\hline & LEE, L.T. et al. & 2018 & Oral Cancer & 41 & $2060.3 \pm$ & Kolmogorov-Smirnov and & $<0.001$ & 0.783 (Sensitivity: \\
\hline
\end{tabular}


Table 1 Salivary interleukin 8 in oral cancer (Continued)

\begin{tabular}{|c|c|c|c|c|c|c|c|c|}
\hline Cytokine & Author & Year & Groups & $\begin{array}{l}\mathrm{n} \text { of } \\
\text { cases }\end{array}$ & $\begin{array}{l}\text { Mean } \pm \\
\text { SD }(p g / \\
\mathrm{ml})\end{array}$ & Statistic test & $p$ Value & AUC \\
\hline & & & & & 1796.5 & \multirow{2}{*}{\multicolumn{2}{|c|}{ Mann-Whitney }} & \multirow{2}{*}{$\begin{array}{l}65.85 \% \\
\text { Specificity: } 79.17 \% \\
p=0.0002)\end{array}$} \\
\hline & & & Control & 24 & $\begin{array}{l}907.0 \pm \\
833.4\end{array}$ & & & \\
\hline & \multirow[t]{3}{*}{$\begin{array}{l}\text { RHODUS, N.L. } \\
\text { et al. }\end{array}$} & $2005 b$ & Oral lichen planus & 13 & $\begin{array}{l}2492.0 \pm \\
664.7\end{array}$ & \multirow[t]{3}{*}{$\begin{array}{l}\text { One-way ANOVA, Student- } \\
\text { Newman- Keuls q-test; t-test. }\end{array}$} & \multirow[t]{3}{*}{$<0.001$} & \multirow[t]{3}{*}{-} \\
\hline & & & Oral Cancer & 13 & $\begin{array}{l}4082.8 \pm \\
752.3\end{array}$ & & & \\
\hline & & & Control & 13 & $\begin{array}{l}1507.2 \pm \\
398.5\end{array}$ & & & \\
\hline & \multirow[t]{2}{*}{ MAIE, A. R. et al. } & \multirow[t]{2}{*}{2004} & Oral Cancer & 32 & 720.0 & \multirow{2}{*}{$\begin{array}{l}\text { t-test, receiver operating } \\
\text { characteristic (ROC) analyses }\end{array}$} & \multirow[t]{2}{*}{$<0.001$} & \multirow{2}{*}{$\begin{array}{l}0.978 \text { (Sensitivity: } \\
86 \% \\
\text { Specificity: 97\%) }\end{array}$} \\
\hline & & & Control & 32 & 250.0 & & & \\
\hline & \multirow{3}{*}{$\begin{array}{l}\text { KHYANI, I.A.M. } \\
\text { et al. }\end{array}$} & \multirow[t]{3}{*}{2017} & Oral Cancer & 35 & 873.6 & \multirow{3}{*}{$\begin{array}{l}\text { Pearson Chi-Square test, } \\
\text { one-way ANOVA test, Post Hoc. } \\
\text { Dunnet t-test }\end{array}$} & \multirow[t]{3}{*}{$<0.001$} & \multirow[t]{3}{*}{-} \\
\hline & & & Control & 35 & 52.1 & & & \\
\hline & & & Premalignant & 35 & 305.0 & & & \\
\hline & \multirow[t]{3}{*}{$\begin{array}{l}\text { RHODUS, N.L. } \\
\text { et al. }\end{array}$} & \multirow[t]{3}{*}{$\begin{array}{l}2005 \\
a\end{array}$} & Oral Cancer & 13 & $\begin{array}{l}3154.1 \pm \\
1023.2\end{array}$ & \multirow[t]{3}{*}{ Not described } & \multirow[t]{3}{*}{$<0.001$} & \multirow[t]{3}{*}{-} \\
\hline & & & Control & 13 & $\begin{array}{l}1580.7 \pm \\
789.0\end{array}$ & & & \\
\hline & & & Premalignant & 13 & $\begin{array}{l}1918.2 \pm \\
899.1\end{array}$ & & & \\
\hline & \multirow[t]{3}{*}{$\begin{array}{l}\text { GLEBER-NETTO, } \\
\text { FO. et al. }\end{array}$} & \multirow[t]{3}{*}{2016} & Oral Cancer & 60 & $\begin{array}{l}283.7 \pm \\
262.3\end{array}$ & \multirow[t]{3}{*}{$\begin{array}{l}\text { ANOVA (Kruskal - Wallis Test); } \\
\text { Wilcoxon Two - Sample test }\end{array}$} & \multirow[t]{3}{*}{$<0.0001$} & \multirow{3}{*}{$\begin{array}{l}\text { OPMD vs. Controls = } \\
0.467 \\
\text { OSCC vs. Controls = } \\
0.449 \\
\text { OSCC vs. OPMD = } \\
0.518\end{array}$} \\
\hline & & & $\begin{array}{l}\text { Malignant } \\
\text { pontentialy injuries }\end{array}$ & 60 & $\begin{array}{l}140.3 \pm \\
155.1\end{array}$ & & & \\
\hline & & & Control & 60 & $\begin{array}{l}127.8 \pm \\
110.8\end{array}$ & & & \\
\hline
\end{tabular}

$26,31,34-38]$, while in two studies it was found in higher values (33.4 and $69.2 \mathrm{pg} / \mathrm{mL}$ ) [19, 39]. The difference in IL-6 salivary concentration between OC patients and healthy individual was also observed in the metaanalysis, that included 12 studies $(\mathrm{SMD}=2.08$; $95 \% \mathrm{CI}$ 1.33 to 2.84$)$. However, heterogeneity was significant $\left(\mathrm{I}^{2}=93 \%, p<0.01\right)$ (Fig. 3).

For the OPMD group, values between 0.431 and 35.3 $\mathrm{pg} / \mathrm{mL}$ were reported in four articles $[20,31,35,37]$ and higher values, between 43.0 and $217.8 \mathrm{pg} / \mathrm{mL}$ were found in another five studies $[18,19,25,26,34]$. In the OC groups, the values were from 0.707 to $435.0 \mathrm{pg} / \mathrm{mL}$ [14, $18-20,22,25,26,31,34-39]$, and the values were higher than $80.0 \mathrm{pg} / \mathrm{mL}$ in nine studies $[14,18-20,26,31,34$, 37, 39]. Meta-analysis showed that IL-6 concentration was significantly higher in OPMD patients in comparison to healthy controls (SMD $=0.97 ; 95 \%$ CI 0.35 to 1.59) (Fig. 4a), while OC patients showed significantly higher IL-6 concentrations in comparison to the OPMD group (SMD $=0.97 ; 95 \%$ CI 1.49 to 0.46 ) (Fig. 4b). In both cases, heterogeneity was high $\left(\mathrm{I}^{2}=85\right.$ and $78 \%$, respectively and $p<0.01$ in both comparisons).

\section{Tumor necrosis factor- $\alpha$ (TNF- $\alpha$ )}

TNF- $\alpha$ was analyzed in 8 articles and it was found in a significantly higher amount in OC patients compared to controls in 7 of these studies. The study population varied from 9 to 100 subjects. Three studies only compared OC patients with a healthy control group [22,39, 40], while 5 articles compared the $\mathrm{OC}$ patients with subjects with OPMD $[20,26,34,35,41]$. The TNF- $\alpha$ values for the control groups were from 0.013 to $11,300.0 \mathrm{pg} / \mathrm{mL}$. For OC patients, the TNF- $\alpha$ values varied from 0.739 to $23,100.0$ $\mathrm{pg} / \mathrm{mL}$ [13, 26, 34, 35, 39-42]. Meta-analysis comparing salivary TNF- $\alpha$ concentration in OC patients against control subjects included seven studies and indicated a significant increase (SMD = 2.04; IC 95\% 0.47 to 3.61) (Fig. 3). However, heterogeneity, as measured by Higgins' $\mathrm{I}^{2}$, reached $97 \%(p<0.01)$. Salivary TNF- $\alpha$ concentrations were not significantly different in OPMD patients in comparison to controls nor in $\mathrm{OC}$ patients in comparison to OPMD (SMD $=2.50 ; 95 \% \mathrm{CI}-0.65$ to 5.65 and $\mathrm{SMD}=$ 0.76 ; $95 \%$ CI -0.11 to 1.64 , respectively). For both summaries, heterogeneity was high $\left(\mathrm{I}^{2}=99\right.$ and $90 \%$, respectively, and $\mathrm{p}<0.01$ in both cases) (Fig. 4a-b). 


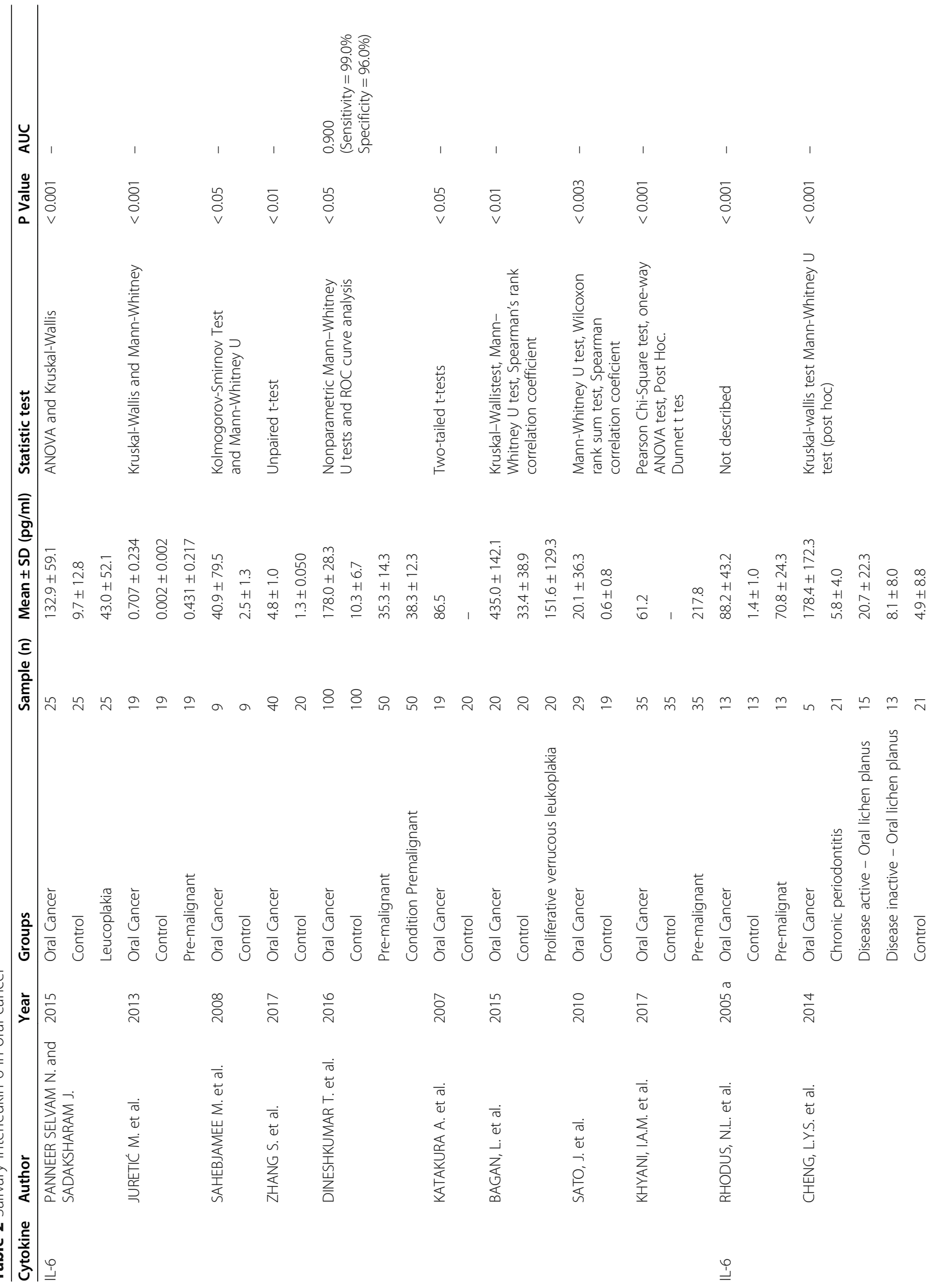




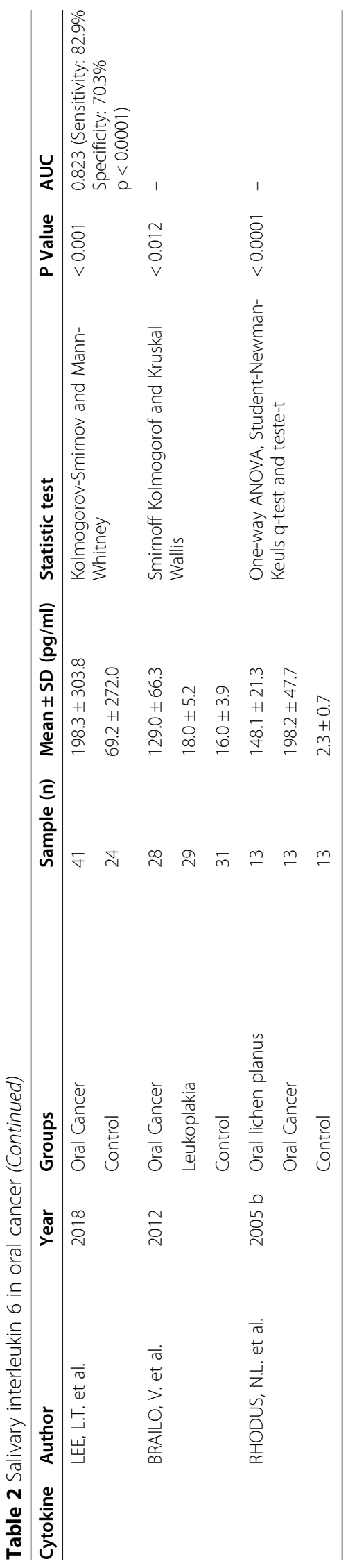




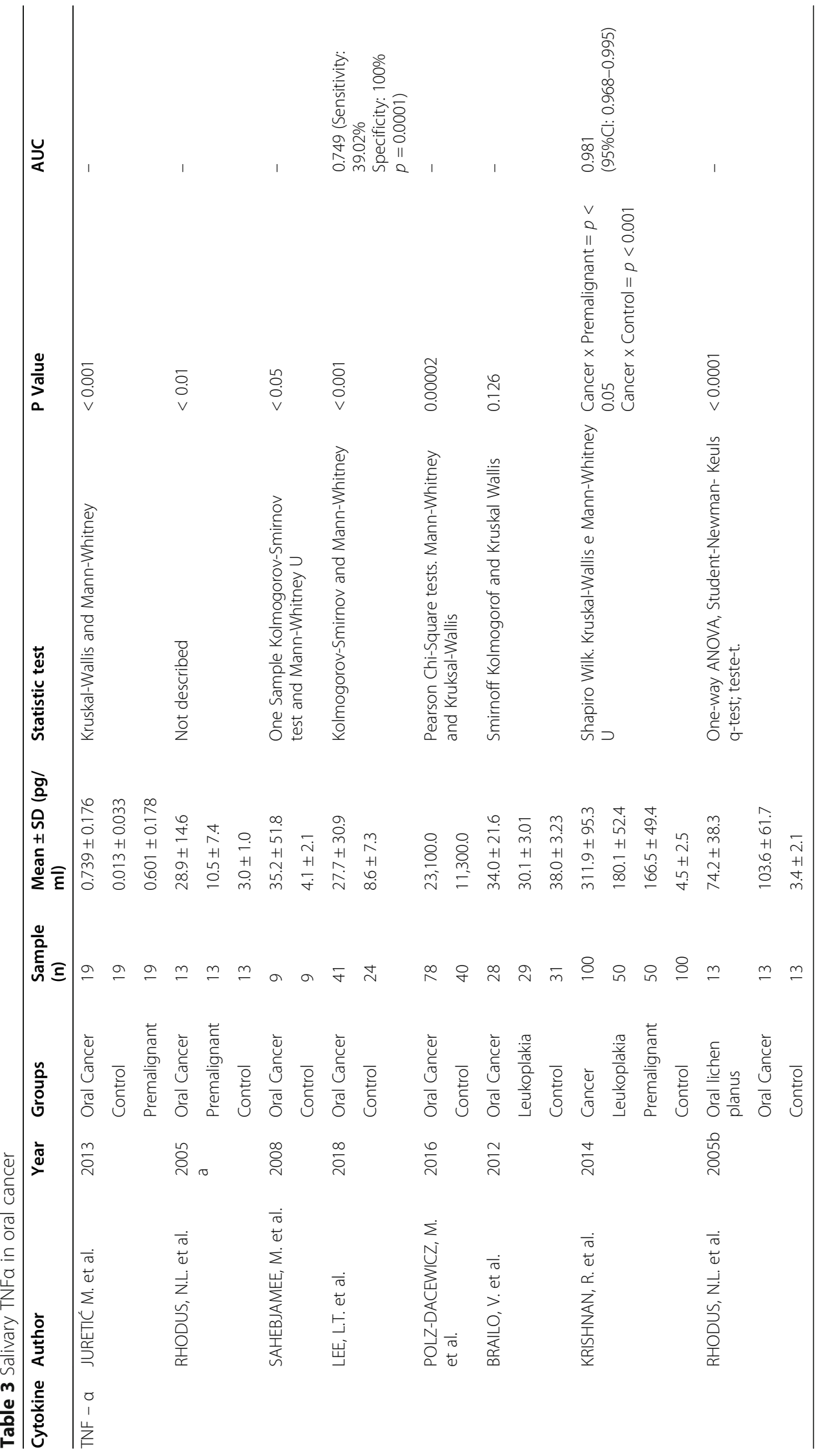


Table 4 Salivary Interleukin $1 \beta$ in oral cancer

\begin{tabular}{|c|c|c|c|c|c|c|c|c|}
\hline Cytokine & Author & Year & Groups & $\begin{array}{l}\text { Sample } \\
\text { (n) }\end{array}$ & Mean \pm SD $(\mathrm{pg} / \mathrm{ml})$ & Statistic test & P Value & AUC \\
\hline \multirow[t]{14}{*}{$\mathrm{IL}-1 \beta$} & \multirow[t]{2}{*}{$\begin{array}{l}\text { ELASHOFF, } \\
\text { D. et al. }\end{array}$} & \multirow[t]{2}{*}{2012} & $\begin{array}{l}\text { Oral Cancer } \\
\text { - cohort } 5\end{array}$ & 31 & $293 \pm 396$ & \multirow{2}{*}{$\begin{array}{l}\text { Mann-Whitney } U \text { test } \\
\text { and area under the } \\
\text { curve (AUC) }\end{array}$} & \multirow[t]{2}{*}{$<0.05$} & \multirow[t]{2}{*}{0.570} \\
\hline & & & $\begin{array}{l}\text { Control - } \\
\text { cohort } 5\end{array}$ & 70 & $169 \pm 202$ & & & \\
\hline & \multirow{2}{*}{$\begin{array}{l}\text { ARELLANO- } \\
\text { GARCIA, M.E. } \\
\text { et al. }\end{array}$} & \multirow[t]{2}{*}{2008} & Oral Cancer & 40 & $591.5 \pm 618.7$ & \multirow{2}{*}{$\begin{array}{l}\text { Student t-test, Pearson } \\
\text { correlation coefficient; } \\
\text { ROC curve and area } \\
\text { under curv }\end{array}$} & \multirow{2}{*}{$\begin{array}{l}\text { With single-plex: } 0.03 \\
\text { With multiplex: } 0.04\end{array}$} & \multirow{2}{*}{$\begin{array}{l}0.840 \\
(\text { sensitivity = 63.9\%; } \\
\text { specificity = 100\%) }\end{array}$} \\
\hline & & & $\begin{array}{l}\text { Control Oral } \\
\text { Cancer }\end{array}$ & 42 & $79.6 \pm 578$ & & & \\
\hline & \multirow{2}{*}{$\begin{array}{l}\text { KATAKURA } \\
\text { A. et al. }\end{array}$} & \multirow[t]{2}{*}{2007} & Oral Cancer & 19 & 158.9 & \multirow[t]{2}{*}{ Two-tailed t-tests } & \multirow[t]{2}{*}{$<0.05$} & \multirow[t]{2}{*}{-} \\
\hline & & & Control & 20 & 14.1 & & & \\
\hline & \multirow{3}{*}{$\begin{array}{l}\text { GLEBER- } \\
\text { NETTO, } \\
\text { FO. et al. }\end{array}$} & \multirow[t]{3}{*}{2016} & Oral Cancer & 60 & $101.0 \pm 113.0$ & \multirow{3}{*}{$\begin{array}{l}\text { ANOVA (Kruskal - Wallis } \\
\text { Test); Wilcoxon Two - } \\
\text { Sample test }\end{array}$} & \multirow{3}{*}{$\begin{array}{l}\text { Oral Cancer } x \\
\text { Control }=p<0.01 ; \\
\text { Oral Cancer } x \\
\text { Maligmant } \\
\text { Potencial }= \\
p<0.004\end{array}$} & \multirow{3}{*}{$\begin{array}{l}\text { OPMD vs. } \\
\text { Controls }=0.542 \\
\text { OSCC vs. } \\
\text { Controls }=0.721 \\
\text { OSCC vs. } \\
\text { OPMD }=0.569\end{array}$} \\
\hline & & & $\begin{array}{l}\text { Malignant } \\
\text { potentialy } \\
\text { injuries }\end{array}$ & 60 & $39.7 \pm 28.0$ & & & \\
\hline & & & Control & 60 & $48.1 \pm 42.0$ & & & \\
\hline & \multirow{2}{*}{$\begin{array}{l}\text { LEE, L.T. } \\
\text { et al. }\end{array}$} & \multirow[t]{2}{*}{2018} & Oral Cancer & 41 & $391.4 \pm 540.4$ & \multirow{2}{*}{$\begin{array}{l}\text { Kolmogorov-Smirnov } \\
\text { and Mann-Whitney }\end{array}$} & \multirow[t]{2}{*}{0.002} & \multirow{2}{*}{$\begin{array}{l}\text { AUC: } 0.729 \\
\text { (Sensitivity =60.98\%; } \\
\text { Specificity = 79.17\%; } \\
p=0.0004 \text { ) }\end{array}$} \\
\hline & & & Control & 24 & $132.5 \pm 175.8$ & & & \\
\hline & \multirow{3}{*}{$\begin{array}{l}\text { BRAILO, V. } \\
\text { et al. }\end{array}$} & \multirow[t]{3}{*}{2012} & Oral Cancer & 28 & $906.0 \pm 62.2$ & \multirow{3}{*}{$\begin{array}{l}\text { Smirnoff Kolmogorof } \\
\text { and Kruskal Wallis }\end{array}$} & \multirow[t]{3}{*}{0.000} & \multirow[t]{3}{*}{-} \\
\hline & & & Leukoplakia & 29 & $143.0 \pm 54.7$ & & & \\
\hline & & & Control & 31 & $354.0 \pm 61.4$ & & & \\
\hline
\end{tabular}

\section{Interleukin-1 $\beta$}

IL- $1 \beta$ was analyzed in 6 articles and a significant difference in salivary concentration was found between OC patients and control subjects in all studies. The number of subjects included in the studies varied from 28 to 60 . This difference was also observed in the meta-analysis, that included five studies (SMD = $0.78 ; 95 \%$ CI 0.44 to 1.13$)$. Heterogeneity between studies was low ( $\mathrm{I}^{2} 65 \%, p=0.02$ ) (Fig. 3). Two studies compared OC patients with both OPMD patients and healthy controls $[20,43]$, and 4 studies compared OC patients with only healthy subjects [23, 39]. The
IL-1 $\beta$ values in the control group varied from 14.1 to $354.0 \mathrm{pg} / \mathrm{mL}$. In the OPMD group the variation was between 39.6 to $143.0 \mathrm{pg} / \mathrm{mL}$, and in the OC patients it varied from 101.0 to $906.0 \mathrm{pg} / \mathrm{mL}[14,23,28,39$, $42,43]$. Meta-analysis showed that IL-1 $\beta$ concentration in OPMD patients was not significantly different from controls with a bordering value, in fact being the only SC showing a tendency of reduction in this condition $(\mathrm{SMD}=-0.40 ; 95 \% \mathrm{CI}-0.80$ to 0.00$)$. Heterogeneity was once again low $\left(\mathrm{I}^{2}=40 \%, \mathrm{p}=0.02\right)$ (Fig. 4a). IL-1 $\beta$ concentration in OC patients in comparison to the OPMD group was not significantly

Table 5 Salivary interleukin 10 in oral cancer

\begin{tabular}{|c|c|c|c|c|c|c|c|c|}
\hline Cytokine & Author & Year & Groups & Sample (n) & $\begin{array}{l}\text { Mean } \pm \text { SD } \\
(\mathrm{pg} / \mathrm{ml})\end{array}$ & Statistic test & P Value & AUC \\
\hline \multirow[t]{10}{*}{$\mathrm{IL}-10$} & \multirow[t]{2}{*}{ HAMZAVI, M. et al. } & \multirow[t]{2}{*}{2014} & Oral Cancer & 30 & $11.8 \pm 10.7$ & \multirow{2}{*}{$\begin{array}{l}\text { Kolmogorov-Smirnov test. Mann-Witney, } \\
\text { Kruskal-Wallis and Chi-Square tests }\end{array}$} & \multirow[t]{2}{*}{0.619} & \multirow[t]{2}{*}{-} \\
\hline & & & Control & 24 & $10.0 \pm 6.0$ & & & \\
\hline & \multirow[t]{2}{*}{ AZIZ, S. et al. } & \multirow[t]{2}{*}{2015} & Oral Cancer & 30 & $4.4 \pm 4.3$ & \multirow[t]{2}{*}{ Teste - t. One way ANOVA e LSD Post hoc } & \multirow[t]{2}{*}{0.004} & \multirow[t]{2}{*}{-} \\
\hline & & & Control & 33 & $1.7 \pm 1.3$ & & & \\
\hline & \multirow[t]{2}{*}{ LEE, L.T. et al. } & \multirow[t]{2}{*}{2018} & Oral Cancer & 41 & $14.9 \pm 20.2$ & \multirow[t]{2}{*}{ Kolmogorov-Smirnov and Mann-Whitney } & \multirow[t]{2}{*}{0.355} & \multirow[t]{2}{*}{-} \\
\hline & & & Control & 24 & $9.9 \pm 8.5$ & & & \\
\hline & \multirow[t]{2}{*}{ POLZ-DACEWICZ, M. et al. } & \multirow[t]{2}{*}{2016} & Oral Cancer & 78 & 5.9 & \multirow{2}{*}{$\begin{array}{l}\text { Pearson Chi-Square tests. } \\
\text { Mann-Whitney and Kruksal-Wallis }\end{array}$} & \multirow[t]{2}{*}{0.00002} & \multirow[t]{2}{*}{-} \\
\hline & & & Control & 40 & 2.5 & & & \\
\hline & \multirow[t]{2}{*}{ GONÇALVES, A.S. et al. } & \multirow[t]{2}{*}{2015} & Oral Cancer & 22 & $0.037^{*}$ & \multirow{2}{*}{$\begin{array}{l}\text { Shapiro-Wilk Mann- Whitney Fisher and } \\
\text { Pearson Chi-Square tests. }\end{array}$} & \multirow[t]{2}{*}{0.038} & \multirow[t]{2}{*}{-} \\
\hline & & & Control & 23 & $0.027^{*}$ & & & \\
\hline
\end{tabular}


Table 6 Salivary interleukin 1a in oral cancer

\begin{tabular}{|c|c|c|c|c|c|c|c|c|}
\hline Cytokine & Author & Year & Groups & Sample (n) & $\begin{array}{l}\text { Mean } \pm \text { SD } \\
(\mathrm{pg} / \mathrm{ml})\end{array}$ & Statistic test & $P$ Value & AUC \\
\hline \multirow[t]{7}{*}{ IL-1a } & \multirow[t]{2}{*}{ SAHEBJAMEE M. et al. } & \multirow[t]{2}{*}{2008} & Oral Cancer & 9 & $201.7 \pm 178.8$ & \multirow{2}{*}{$\begin{array}{l}\text { One Sample Kolmogorov-Smirnov } \\
\text { test and Mann-Whitney U }\end{array}$} & \multirow[t]{2}{*}{$<0.05$} & \multirow[t]{2}{*}{-} \\
\hline & & & Control & 9 & $178.2 \pm 170.7$ & & & \\
\hline & \multirow[t]{2}{*}{ LEE, L.T. et al. } & \multirow[t]{2}{*}{2018} & Oral Cancer & 41 & $995.7 \pm 932.6$ & \multirow{2}{*}{$\begin{array}{l}\text { Kolmogorov-Smirnov and Mann- } \\
\text { Whitney }\end{array}$} & \multirow[t]{2}{*}{0.625} & \multirow[t]{2}{*}{-} \\
\hline & & & Control & 24 & $1054.6 \pm 1584.9$ & & & \\
\hline & \multirow[t]{3}{*}{ RHODUS, N.L. et al. } & \multirow[t]{3}{*}{$2005 b$} & Oral lichen planus & 13 & $293.6 \pm 86.8$ & \multirow{3}{*}{$\begin{array}{l}\text { One-way ANOVA, Student-Newman- } \\
\text { Keuls q-test; teste-t. }\end{array}$} & \multirow[t]{3}{*}{$<0.001$} & \multirow[t]{3}{*}{-} \\
\hline & & & Oral Cancer & 13 & $370.5 \pm 29.7$ & & & \\
\hline & & & Control & 13 & $135.9 \pm 28.4$ & & & \\
\hline
\end{tabular}

different (SMD = 1.55; IC 95\% -0.09 to 3.18 ) and with high heterogeneity $\left(\mathrm{I}^{2}=94 \%, p<0.01\right)$ (Fig. 4b).

\section{Interleukin-10}

IL-10 was evaluated in 5 articles and in three of them was observed statistical difference in OC patients compared do control subjects. The studies evaluated from 22 to 78 subjects per group. All articles compared OC patients to control subjects, not including OPMD patients. The salivary IL-10 values were between 0.027 and 10.0 $\mathrm{pg} / \mathrm{mL}$ in control groups, and between 0.037 and 14.9 $\mathrm{pg} / \mathrm{mL}$ in OC patients [39, 40, 44-46]. Meta-analysis included three studies, and the combined effect indicates an increase in the salivary concentration of IL-10 in OC patients in comparison to healthy subjects (SMD $=0.46$; $95 \%$ CI 0.05 to 0.86$)$. Heterogeneity of this analysis was low $\left(\mathrm{I}^{2}=46 \%, p=0.16\right)$ (Fig. 3 ).

\section{Interleukin-1a}

IL- $1 \alpha$ was evaluated in 3 articles, two compared OC patients with healthy controls [22, 39] and one study also compared with OLP [34] (Fig. 3). The studies included from 9 to 41 subjects per group. Two of these articles presented results with significant difference between $\mathrm{OC}$ and controls. The IL- $1 \alpha$ quantification varied from 135.9 to $1054.6 \mathrm{pg} / \mathrm{mL}$ in the control groups, was of $293.6 \mathrm{pg} /$ $\mathrm{mL}$ in the OLP group, and between 201.7 and $995.7 \mathrm{pg} /$ $\mathrm{mL}$ in $\mathrm{OC}$ patients [22, 34, 39]. Results from metaanalysis showed a non-significant difference between salivary levels of IL- $1 \alpha$ of OC patients in comparison to controls $(\mathrm{SMD}=2.21 ; 95 \% \mathrm{CI}-0.36$ to 4.77$)$, even though the analysis had a high heterogeneity $\left(\mathrm{I}^{2}=95 \%\right.$, $p<0.01$ ) (Fig. 3).

\section{Other cytokines}

Due to the lack of studies to draw comparisons, the remaining cytokines were included only in a qualitative analysis.

IL-1RA was found in one study that compared OC patients $(n=30)$ with healthy controls $(n=33)$ (Fig. 3). The salivary concentration of IL-1RA in the OC group was of $2831.6 \mathrm{pg} / \mathrm{mL}$, and $1949.2 \mathrm{pg} / \mathrm{mL}$ in control group, not showing significant difference between them [46].

IL-1 (without specification of the subunit) was studied in one article, comparing OC patients with patients with OPMD and a group of healthy controls (Fig. 3), each group was composed of 13 individuals. Differences were found in the concentration of salivary IL-1 between groups. The IL-1 value found in OC patients was of $454.4 \mathrm{pg} / \mathrm{mL}$, in premalignant lesions was of $255.1 \mathrm{pg} /$ $\mathrm{mL}$, and, in healthy controls, of $173.2 \mathrm{pg} / \mathrm{mL}$ [26].

IL-4 was studied in one article comparing OC patients $(\mathrm{n}=30)$ with healthy subjects $(\mathrm{n}=33)$ (Fig. 3$)$. OC patients present $1.2 \mathrm{pg} / \mathrm{mL}$ of IL- 4 in saliva, while healthy subjects $1.0 \mathrm{pg} / \mathrm{mL}$, without statistical difference between groups [46].

IL-13 also was analyzed in one study, comparing the same groups as the previous one (Fig. 3). In OC patients the IL-13 level was $0.760 \mathrm{pg} / \mathrm{mL}$, and in the control groups $0.230 \mathrm{pg} / \mathrm{mL}$, showing a difference statistically significant [46].

\section{Discussion}

Pro-inflammatory and anti-inflammatory cytokines are produced and released in the tumor microenvironment by tumor and immune cells [47]. Studies with saliva have shown increased cytokine levels in cancer patients, irrespective of pro-inflammatory or anti-inflammatory cytokine activity [33, 48].

In the studies analyzed in this review different proinflammatory and anti-inflammatory cytokines were evaluated (pro-inflammatory: IL-1, IL-1 $\alpha$, IL-1 $\beta$, IL-RA, IL-6, IL-8 and TNF $\alpha$; anti-inflammatory: IL-4, IL-10 e IL-13). The most studied cytokines were IL-8 and IL-6. The salivary levels of IL- 8 , IL-6, TNF- $\alpha$, IL- $1 \beta$ and IL- $1 \alpha$ were significantly higher in $\mathrm{OC}$ patients compared to healthy controls. Only one anti-inflammatory SC (IL-10) was evaluated by meta-analysis, and did not presented significant difference between $\mathrm{OC}$ and NCC. Furthermore, the levels of salivary IL- 8 , IL-6, TNF- $\alpha$ and IL- $1 \beta$ were higher in $\mathrm{OC}$ patients compared to PMOL, and comparing the NCC vs PMOL patients, was observed a 

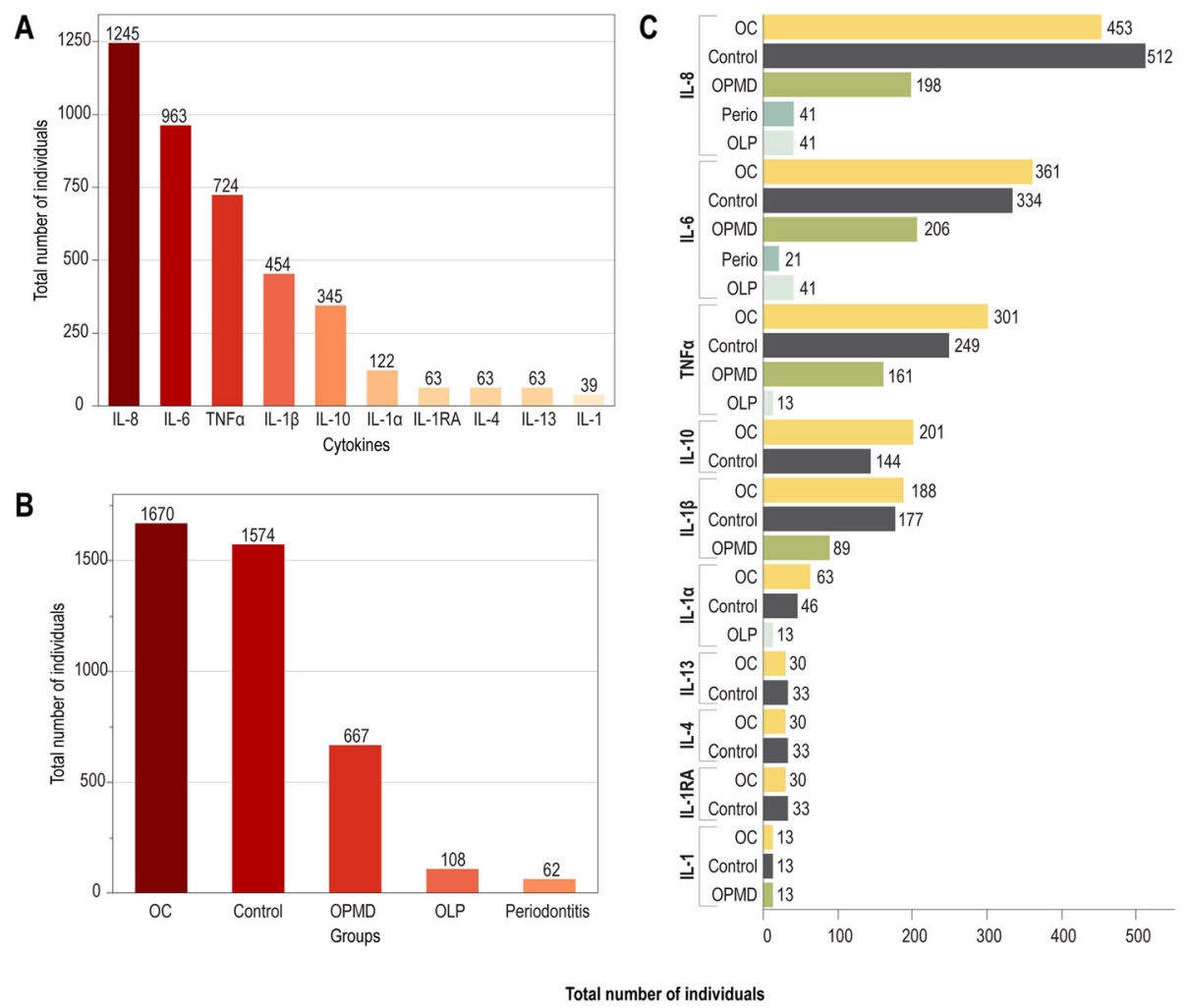

Total number of individuals

Fig. 2 Sample size distribution among the investigated studies according to: A) Salivary cytokines assessed. IL-8, IL-6, TNFa and IL-1 $\beta$ were the cytokines investigated in the biggest number of patients; B) Patient clinical groups (OC = Oral Cancer; Control $=$ healthy individuals; OPMD = Oral Potentially Malignant Disorders, OLP = Oral Lichen Planus). Healthy patients were the most numerous among control groups, followed by individuals with OPMD; C) Salivary cytokines assessed per each patient clinical group

significant increasing in salivary levels of IL-6 and TNF$\alpha$ in PMOL group.

Our study showed that some SC show great potential as diagnostic biomarkers for $\mathrm{OC}$, serving as a noninvasive alternative for early diagnosis. The increased concentration of certain SC, most notably IL-8, IL-6 and TNF- $\alpha$, could be used as biomarkers for OC, as their concentration was significantly higher in patients with the disease in comparison to healthy subjects. However, the levels of these $\mathrm{SC}$ are also increased due to other conditions, such as OPMD. Thus, it is important to determine how different is this increase when comparing the two clinical conditions. It was observed that the concentration of IL- 8 and IL- 6 in OC patients was significantly higher in comparison to OPMD patients, even with a significant increase in comparison to healthy subjects, making these two of the most promising $\mathrm{SC}$ as reliable diagnostic tools. None of the other SC included in the meta-analysis showed a clear difference in concentration between OC and OPMD patients, even though differences were observed when comparing patients with
OC patients and healthy subjects, (namely, TNF- $\alpha$, IL- $1 \beta$ and IL-10). This suggests good potential to determine $\mathrm{OC}$, even though evidence for these $\mathrm{SC}$ is more limited.

There are still technical and biological issues that must be addressed for a final and definitive evaluation of the clinical value of these biomarkers. Even though all studies analyzed in this review used the ELISA, considered as the most accurate technique to measure proteins in biofluids [16], one of the main limitation of the results is the high heterogeneity of the results collected, even in the control groups. Even though this variability limits the interpretation of the meta-analysis, it favors the discussion about the development and implementation of standard operating procedures (SOP) in the field of salivary biomarkers, leading to its technical development and facilitating its translational path. With more standardized methods of saliva collection and storage and SC quantification, thresholds could be identified to distinguish between different oral conditions.

Even though the studies compared patients with OC with different control groups, from healthy subjects to 
Study

$\mathbf{s C}=\mathrm{IL}-8$

Arellano-Garcia et al. (2008)

Cheng et al. (2014)

Elashoff et al. (2012)

Elashoff et al. (2012)

Gleber-Netto et al. (2016)

Lee et al. (2018)

Punyani and Sathawane (2013)

Rajkumar et al. (2014)

Rhodus et al. (2005a)

Rhodus et al. (2005b)

Sahebjamee et al. (2008)

Tann et al. (2007)

Summary

$l^{2}=82 \%, \tau^{2}=0.3405, p<0.01$

$\mathbf{S C}=\mathrm{IL}-6$

Bagan et al. (2015)

Brailo et al. (2012)

Cheng et al. (2014)

Dineshkumar et al. (2011)

Juretic et al. (2013)

Lee et al. (2018)

Panneer Selvam and Sadaksharam (2015)

Rhodus et al. (2005a)

Rhodus et al (2005b)

Sahebjamee et al. (2008)

Sato et al. (2010)

Zhang et al. (2017)

Summary

$l^{2}=93 \%, \tau^{2}=1.5754, p<0.01$

$\mathrm{sc}=\mathrm{TNF} \alpha$

Brailo et al. (2012)

Juretic et al. (2013)

Krishnan et al. (2014)

Lee et al. (2018)

Rhodus et al. (2005a)

Rhodus et al. (2005b)

Sahebjamee et al. (2008)

Summary

$l^{2}=97 \%, \tau^{2}=4.2953, p<0.01$

Sc $=\mathrm{IL}-1 \beta$

Arellano-Garcia et al. (2008)

Brailo et al. (2012)

Elashoff et al. (2012)

Gleber-Netto et al. (2016)

Lee et al. (2018)

Summary
$l^{2}=65 \%, \tau^{2}=0.0999, p=0.02$

$\mathbf{S c}=\mathrm{IL}-10$

Aziz et al. (2015)

Hamzavi et al. (2014)

Lee et al. (2018)

Summary

$l^{2}=46 \%, \tau^{2}=0.0596, p=0.16$

sc $=\mathrm{IL}-1 \alpha$

Lee et al. (2018)

Rhodus et al. (2005b)

Sahebjamee et al. (2008)

Summary

$l^{2}=95 \%, \tau^{2}=4.6029, p<0.01$
SMD $\quad 95 \%-\mathrm{Cl}$ Weight

$1.23[0.76 ; 1.70] \quad 8.9 \%$

$0.89[-0.12 ; 1.90] \quad 7.5 \%$

$0.92[0.48 ; 1.36] \quad 9.0 \%$

$1.07[0.62 ; 1.52] \quad 9.0 \%$

$0.77[0.40 ; 1.14] \quad 9.1 \%$

$0.75[0.23 ; 1.27] \quad 8.8 \%$

$3.07[2.24 ; 3.91] \quad 8.0 \%$

$0.52[0.23 ; 0.80] \quad 9.2 \%$

$0.46[-0.32 ; 1.24] \quad 8.2 \%$

$4.14[2.70 ; 5.59] \quad 6.2 \%$

$0.35[-0.58 ; 1.29] \quad 7.7 \%$

$\begin{array}{rrr}-\quad & 1.62[0.90 ; 2.34] & 8.3 \% \\ -\quad 1.17 & \end{array}$

$\begin{array}{rrr}- & 1.62[0.90 ; 2.34] & 8.3 \% \\ - & \mathbf{1 . 1 7}[0.79 ; \mathbf{1 . 5 5}] \mathbf{1 0 0 . 0 \%}\end{array}$

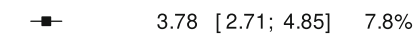

$0.46[-0.06 ; 0.98] \quad 9.4 \%$

$2.37[1.17 ; 3.57] \quad 7.4 \%$

$0.81[0.52 ; 1.10] \quad 9.9 \%$

$4.17[2.99 ; 5.35] \quad 9.9 \%$

7.5\%

$0.44[-0.07 ; 0.95] \quad 9.5 \%$

$2.84[2.03 ; 3.64] \quad 8.7 \%$

$0.76[-0.04 ; 1.56] \quad 8.7 \%$

$5.63[3.80 ; 7.46] \quad 5.5 \%$

$0.65[-0.30 ; 1.61] \quad 8.2 \%$

$0.68 \quad[0.08 ; 1.27] \quad 9.3 \%$

$4.21[3.26 ; 5.16] \quad 8.2 \%$

$2.08[1.33 ; 2.84] 100.0 \%$

$-0.05[-0.56 ; 0.46] \quad 15.9 \%$ $5.61[4.14 ; 7.09] 10.9 \%$ $4.54[4.01 ; 5.07] 15.8 \%$ $0.76 \quad[0.23 ; 1.28] 15.8 \%$ $0.67[-0.12 ; 1.47] \quad 14.6 \%$ $2.23[1.21 ; 3.24] \quad 13.4 \%$ $0.81[-0.16 ; 1.78]-13.4 \%$ $2.04[0.47 ; 3.61] 100.0 \%$

$0.85[0.39 ; 1.30] \quad 20.1 \%$ $1.62[1.03 ; 2.22] \quad 19.4 \%$ $0.45[0.02 ; 0.87] 20.2 \%$ $0.62[0.25 ; 0.98] 20.5 \%$ $0.58[0.06 ; 1.09] 19.8 \%$

$0.78[0.44 ; 1.13] 100.0 \%$

$0.87[0.35 ; 1.39] \quad 33.4 \%$ $0.20[-0.34 ; 0.74] 33.2 \%$ $0.30[-0.21 ; 0.80] \quad 33.5 \%$ $0.46[0.05 ; 0.86] 100.0 \%$

$0.05[-0.55 ; 0.46] \quad 43.5 \%$ $7.82[5.39 ; 10.26] 18.5 \%$

$0.13[-0.80 ; 1.05] \quad 38.0 \%$

$2.21[-0.36 ; 4.77] 100.0 \%$

Fig. 3 Random-effects meta-analysis of the salivary cytokine levels in oral cancer patients in comparison to healthy controls $(S M D=0)$

OPMD and inflammatory conditions, the selection of non-cancer individuals was heterogeneous between the studies, making difficult to carry out direct comparisons between them. This is another point to be observed and standardized in future studies, since the demographic and clinical characteristics of the controls and diseaseinflicted subjects must show correspondence.
Although the relative expression of SC demonstrated a good potential to distinguish OC patients from non-cancer subjects, and the increased levels of these cytokines in $\mathrm{OC}$ patients are consistent, the salivary biomarkers should be tested in combination with clinical examination [49]. The use of these biomarkers must be improved to be used specifically in 

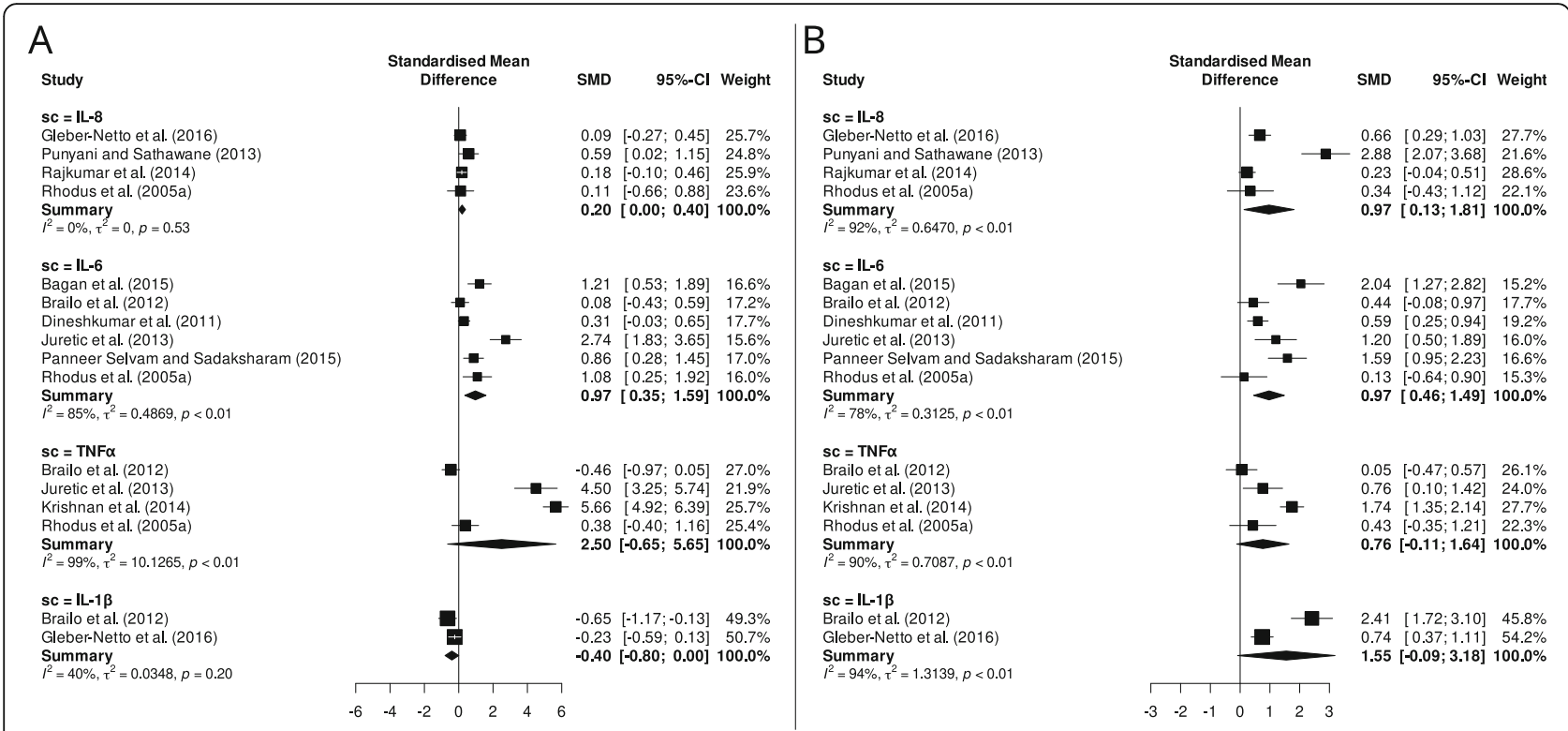

Fig. 4 Random-effects meta-analysis of salivary cytokine levels in patients with oral potentially malignant disorders (OPMD) in comparison to healthy controls (a) and in patients with oral cancer in comparison to individuals with OPMD (b). In both cases, SMD $=0$ for the second group referred

at-risk populations as an auxiliary method for screening and early diagnosis.

An important limitation of the studies we evaluated is the absence of a group of early stage OC patients. Most studies did not separate patients according to disease stage, which limits the extrapolation of these findings to early cancer diagnosis [50]. Some studies showed a positive correlation between cytokine levels disease stage [33]. In this way, it is important that the diagnostic performance of SC is evaluated in the group with early stage disease.

Another limitation is the ELISA kits used to measure the cytokines in these studies, which are reagents label as "Research Use Only", indicating that there is no strict regulation on the technical characteristics of the tests. Thus, the comparison of results between different studies is limited, since the variation between brands or even lots can lead to variations in results. For the continuity of the validation process of these $\mathrm{SC}$ as biomarkers for OC it is necessary the development of studies that consider the variability of the disease presentation, the measurement of the cytokines in a controlled environment, and using reagents developed to clinical use. Moreover, so that possible biases are excluded, multicenter studies must be performed, using a larger samplez than the previous studies $[15,51]$. And for the results to be reliable, it is of fundamental importance that an international standardization be validated for both saliva collection and SC measurements.

\section{Conclusions}

In this review, we found 28 articles that evaluated the concentration of 10 different pro and anti-inflammatory SC in OC patients. IL- 8 and IL- 6 were the most studied ones, and in all articles these salivary cytokines were found at higher levels in OC patients compared to healthy controls and, in most cases, OPMD patients.

A meta-analysis with twenty-three studies showed that salivary levels of IL-8, IL-6, TNF- $\alpha$, IL- $1 \beta$ and IL- $1 \alpha$ are significantly higher in OC patients compared to healthy controls; the levels of salivary IL- 8 and IL- 6 are higher in OC patients compared to OPMD, and comparing the controls vs OPMD patients, it was observed a significant increase in salivary levels of IL-6 in OPMD group. These results suggest that, mainly the pro-inflammatory cytokines IL- 8 and IL- 6 can be explored in the future to determine the real potential as a biomarker for OC. Furthermore, it was found a big variability in the SC concentrations in the different studies, even when reporting the same quantification methodology.

In order to translate these biomarkers into the clinical practice, standardization of saliva collection and cytokines measurement process is required, as well as larger and multicentric studies.

\section{Abbreviations}

SC: Salivary cytokines; OC: Oral squamous cell carcinoma; OPMD: oral potentially malignant disorders; ELISA: enzyme-linked immunosorbent assay; OLP: oral lichen planus; IL-8: interleukin-8; IL-6: Interleukin-6; TNF-a: Tumor necrosis factora; IL-1 $\beta$ : Interleukin-1 $\beta$; IL-10: Interleukin-10; IL-1a: Interleukin- 
1a; IL-1RA: Interleukin-1RA; IL-4: Interleukin-4; IL-13: Interleukin-13; SMD: standardized mean difference; SOP: standard operating procedures

\section{Acknowledgements}

Not Applicable.

\section{Authors' contributions}

All Authors read and approved the manuscript. MMAC: Study concept and design; analysis and interpretation of data; drafting of the manuscript. CBZ: Study concept and design; analysis and interpretation of data; drafting of the manuscript. APR: Drafting of the manuscript; critical revision of the manuscript. MFC: Meta-analysis and critical revision of the manuscript. FOGN: Study concept and design; analysis and interpretation of data; drafting of the manuscript; critical revision of the manuscript. ARB: Study concept and design; analysis and interpretation of data; drafting of the manuscript; critical revision of the manuscript.

\section{Funding}

No funding was received.

\section{Availability of data and materials}

All data generated or analyzed during this study are available for consultation and can be requested from authors.

\section{Ethics approval and consent to participate}

Not applicable.

\section{Consent for publication}

Not applicable.

\section{Competing interests}

The authors declare that they have no conflict of interest.

\section{Author details}

${ }^{1}$ Universidade do Oeste de Santa Catarina (UNOESC), Joacaba, SC, Brazil. ${ }^{2}$ Programa de Pos-Graduacao em Biociencias e Saude/Universidade do Oeste de Santa Catarina, Joacaba, SC, Brazil. ${ }^{3}$ Hospital Universitario Santa Terezinha, Joacaba, SC, Brazil. ${ }^{4}$ Department of Head and Neck Surgery, The University of Texas MD Anderson Cancer Center, Houston, TX, USA.

Received: 17 July 2020 Accepted: 18 February 2021

\section{Published online: 27 February 2021}

\section{References}

1. Siegel RL, Miller KD, Jemal A. Cancer statistics, 2019. CA Cancer J Clin. 2019; 69:7-34. https://doi.org/10.3322/caac.21551.

2. Amit M, Yen TC, Liao CT, Binenbaum Y, Chaturvedi P, Agarwal JP, et al. Clinical nodal stage is a significant predictor of outcome in patients with oral cavity squamous cell carcinoma and pathologically negative neck metastases: results of the international consortium for outcome research. Ann Surg Oncol. 2013;20:3575-81. https://doi.org/10.1245/s1 0434-013-3044-0.

3. Curado MP, Johnson NW, Kerr AR, Pereira L, Mendonc DR, Lanfranchi H. Oral and oropharynx cancer in South America : Incidence, mortality trends and Oral and oropharynx cancer in South America : Incidence, mortality trends and gaps in public databases as presented to the Global Oral Cancer Forum. Transl Res Oral Oncol. 2016;1:1-7.

4. GANESH D, SREENIVASAN P, ÖHMAN J, WALLSTRÖM M, BRAZ-SILVA PH, GIGLIO D, et al. Potentially malignant Oral disorders and Cancer transformation. Anticancer Res. 2018:38:3223-9.

5. Napier SS, Speight PM. Natural history of potentially malignant oral lesions and conditions: an overview of the literature. J Oral Pathol Med. 2008;37:110. https://doi.org/10.1111/j.1600-0714.2007.00579.x.

6. Dionne KR, Warnakulasuriya S, Binti Zain R, Cheong SC. Potentially malignant disorders of the oral cavity: Current practice and future directions in the clinic and laboratory. Int J Cancer. 2014;.n/a-n/a. doi:https://doi.org/1 $0.1002 /$ ijc. 28754

7. Warnakulasuriya S. Causes of oral cancer - an appraisal of controversies. Br Dent J. 2009;207:471-5. https://doi.org/10.1038/sj.bdj.2009.1009.

8. Bagan J, Sarrion G, Jimenez Y. Oral cancer: Clinical features. Oral Oncol. 2010;46:414-7. https://doi.org/10.1016/j.oraloncology.2010.03.009.
9. Neville BW, Day TA. Oral cancer and precancerous lesions, CA Cancer J Clin. 52:195-215. https://doi.org/10.3322/canjclin.52.4.195.

10. Chang K, Chang Y, Liao C, Yen T, Chen I, Chang Y. Clinica Chimica Acta prognostic cytokine markers in peripheral blood for oral cavity squamous cell carcinoma identi fi ed by multiplexed immunobead-based pro fi ling. Clin Chim Acta. 2011:412:980-7.

11. Tiwari M. Science behind human saliva. J Nat Sci Biol Med. 2011;2:53. https://doi.org/10.4103/0976-9668.82322.

12. Schafer CA, Schafer JJ, Yakob M, Lima P, Camargo P, Wong DTW. Saliva Diagnostics: Utilizing Oral Fluids to Determine Health Status. 2014. p. 88-98

13. Sahebjamee M, Eslami M, Atarbashimoghadam F, Sarafnejad A. Salivary concentration of TNF??, IL1??, IL6, and IL8 in oral squamous cell carcinoma. Med Oral Patol Oral Cir Bucal. 2008;13:292-5.

14. Katakura A, Kamiyama I, Takano N, Shibahara T, Muramatsu T, Ishihara K, et al. Comparison of salivary cytokine levels in Oral Cancer patients and healthy subjects. Bull Tokyo Dent Coll. 2007;48:199-203.

15. Osman TA, Costea DE, Johannessen AC. The use of salivary cytokines as a screening tool for oral squamous cell carcinoma : a review of the literature. J Oral Maxillofac Pathol. 2012;16:256-61.

16. Prasad G, McCullough M. Chemokines and cytokines as salivary biomarkers for the early diagnosis of oral cancer. Int J Dent. 2013;2013:813756.

17. Sahibzada HA, Khurshid Z, Khan RS, Naseem M, Siddique KM, Mali M, et al. Salivary IL-8, IL-6 and TNF-a as Potential Diagnostic Biomarkers for Oral Cancer. Diagnostics (Basel, Switzerland). 2017;7. https://doi.org/10.3390/dia gnostics7020021.

18. Selvam NP, Sadaksharam J. Salivary interleukin-6 in the detection of oral cancer and precancer. Asia-Pacific J Clin Oncol. 2015;2015(11):236-41.

19. Bagan L, Sáez GT, Tormos MC, Labaig-rueda C, Murillo-cortes J, Bagan JV. Salivary and serum interleukin-6 levels in proliferative verrucous leukoplakia. Clin Oral Invest DOI. 2015.

20. Brailo V, Vucicevic-Boras V, Lukac J, Biocina-Lukenda D, Zilic-Alajbeg I, Milenovic A, et al. Salivary and serum interleukin 1 beta, interleukin 6 and tumor necrosis factor alpha in patients with leukoplakia and oral cancer. Med Oral Patol Oral Cir Bucal. 2012;17.

21. Green S, Higgins J (editors). Preparing a Cochrane review. In: Green S, Higgins J (editors)., editors. Cochrane Handbook for Systematic Reviews of Interventions. Version 5.1.0. The Cochrane Collaboration; 2011. www.cochra ne-handbook.org.

22. SahebJamee M, Eslami M, AtarbashiMoghadam F, Sarafnejad A. Salivary concentration of TNFalpha, IL1 alpha, IL6, and IL8 in oral squamous cell carcinoma. Med Oral Patol Oral Cir Bucal. 2008;13:E292-5.

23. Elashoff D, Zhou H, Reiss J, Wang J, Henson B, Hu S, Arellano M, Sinha U, Le A, Messadi D, Wang M, Nabili V, Lingen M, Morris D, Randolph T, Ziding Feng DA. Pre-Validation of Salivary Biomarkers for Oral Cancer Detection. Cancer Epidemiol Biomarkers Prev. 2012;21:664-72.

24. St. John MAR, Li Y, Zhou X, Denny P, Ho C-M, Montemagno C, Shi W, Qi F, Wu B, Sinha U, Jordan R, Wolinsky L. Interleukin 6 and interleukin 8 as potential biomarkers for Oral cavity and Oropharyngeal squamous cell carcinoma. Arch Otolaryngol Head Neck Surg. 2004;130:929-35.

25. Khyani IAM, Qureshi MA, Mirza T, Farooq MU. Detection of interleukins-6 and 8 in saliva as potential biomarkers of oral pre-malignant lesion and oral carcinoma : a breakthrough in salivary diagnostics in Pakistan. Pak J Pharm Sci. 2017;30:817-23.

26. Rhodus NL, Ho V, Miller CS, Myers S, Ondrey F. NF-KB dependent cytokine levels in saliva of patients with oral preneoplastic lesions and oral squamous cell carcinoma. Cancer Detect Prev. 2005:29:42-5.

27. Gleber-Netto FO, Yakob M, Li F, Feng Z, Dai J, Kao HK, et al. Salivary biomarkers for detection of oral squamous cell carcinoma in a Taiwanese population. Clin Cancer Res. 2016;22:3340-7.

28. Arellano-Garcia ME, Hu S, Wang J, Henson B, Zhou H, Chia D, D W. Multiplexed immunobead-based assay for detection of oral cancer protein biomarkers in saliva. Oral Dis. 2008;14:705-12.

29. Tan W, Sabet L, Li Y, Yu T, Klokkevold PR, David T. Optical protein sensor for detecting Cancer markers in saliva. Biosens Bioelectron. 2008 24:266-71.

30. Rajkumar K, Nandhini G, Ramya R, Rajashree P, Sc M, Kumar AR, et al. Validation of diagnostic utility of salivary interleukin-8 in differentiation of potentially malignant oral lesion and malignant oral squamous cell carcinoma in high endemic setting region. Oral Surg Oral Med Oral Pathol Oral Radiol. 2014. 
31. Lisa Cheng Y-S, Jordan L, Gorugantula LM, Schneiderman E, Chen H-S, Rees T. Salivary Interleukin-6 and -8 in patients with Oral Cancer and patients with chronic Oral inflammatory diseases. J Periodontol. 2014;85:956-65.

32. Punyani SR, Sathawane RS. Salivary level of interleukin-8 in oral precancer and oral squamous cell carcinoma. Clin Oral Investig. 2013;17:517-24.

33. Lee LT, Wong YK, Hsiao HY, Wang YW, Chan MY, Evaluation KWC. Evaluation of saliva and plasma cytokine biomarkers in patients with oral squamous cell carcinoma. Int J Oral Maxillofac Surg. 2017.

34. Rhodus NL, Cheng B, Myers S, Miller L, Ho V, Ondrey F. The feasibility of monitoring NF-??B associated cytokines: TNF-??, IL-1??, IL-6, and IL-8 in whole saliva for the malignant transformation of oral lichen planus. Mol Carcinog. 2005:44:77-82.

35. Juretić M, Cerović R, Belušić-Gobić M, Pršo IB, Kqiku L, SP-Ribari SŠ. Salivary Levels of TNF- $a$ and IL-6 in Patients with Oral Premalignant and Malignant Lesions. Folia Biol. 2013;59:99-102.

36. Zhang S, Zhang X, Yin K, Li T, Bao Y, Chen Z. Variation and significance of secretory immunoglobulin???A, interleukin 6 and dendritic cells in oral cancer. Oncol Lett. 2017;13:2297-303.

37. Dineshkumar T, Ashwini BK, Rameshkumar A, Rajashree P, Ramya R, Rajkumar K. Salivary and serum Interleukin-6 levels in Oral premalignant disorders and squamous cell carcinoma: diagnostic value and Clinicopathologic correlations. Asian Pac J Cancer Prev. 2016;17:4899-906.

38. Sato J, Goto J, Murata T, Kitamori S. Changes in saliva interleukin-6 levels in patients with oral squamous cell carcinoma. Oral Surg Oral Med Oral Pathol Oral Radiol Endod. 2010;110:330-6.

39. Lee LT, Wong YK, Hsiao HY, Wang YW, Chan MY, Chang KW. Evaluation of saliva and plasma cytokine biomarkers in patients with oral squamous cell carcinoma. Int J Oral Maxillofac Surg. 2018;47:699-707.

40. Polz-dacewicz M, Strycharz-dudziak M, Dworza J, Stec A. VEGF levels in oropharyngeal squamous cell carcinoma and correlation with HPV and EBV infections. Infect Agent Cancer. 2016;11:1-8.

41. Krishnan R, Thayalan DK, Padmanaban R, Ramadas R, Annasamy RK, Anandan N. Association of serum and salivary tumor necrosis factor-a with histological grading in oral cancer and its role in differentiating premalignant and malignant oral disease. Asian Pacific J Cancer Prev. 2014;15:7141-8.

42. Brailo V, Vucicevic-Boras V, Lukac J, Biocina-Lukenda D, Zilic-Alajbeg I, Milenovic A, et al. Salivary and serum interleukin 1 beta, interleukin 6 and tumor necrosis factor alpha in patients with leukoplakia and oral cancer. Med Oral Patol Oral Cir Bucal. 2012;17:e10-5.

43. Gleber-netto FO, Yakob M, Li F, Feng Z, Dai J, Kao H, et al. Salivary biomarkers for detection of oral squamous cell carcinoma in a Taiwanese population. Clin Cancer Res. 2016;22:3340-7.

44. Gonçalves AS, Arantes DAC, Bernardes VF, Jaeger F, Silva JM, Silva TA, et al. Immunosuppressive mediators of oral squamous cell carcinoma in tumour samples and saliva. Hum Immunol. 2015;76:52-8.

45. Hamzavi M, Tadbir AA, Rezvani G, Ashraf J, Fattahi MJ, Khademi B, et al. Tissue Expression, Serum and Salivary Levels of IL-10 in Patients with Head and Neck Squamous Cell Carcinoma. Asian Pacific J Cancer Prev. 2013;14:1681-5.

46. Aziz S, Ahmed SS, Ali A, Khan FA, Zulfiqar G, lqbal J, et al. Salivary immunosuppressive cytokines IL-10 and IL-13 are significantly elevated in Oral squamous cell carcinoma patients. Cancer Investig. 2015;33:318-28.

47. Onuchic AC, Chammas R. Câncer e o microambiente tumoral. Rev Med. 2010;89:21.

48. Jiang C, Ye D, Qiu W, Zhang X, Zhang Z, He D, et al. Response of lymphocyte subsets and cytokines to Shenyang prescription in SpragueDawley rats with tongue squamous cell carcinomas induced by $4 \mathrm{NQO}$. BMC Cancer. 2007;7:40

49. SCIUBBA JJ. Oral cancer and its detection. J Am Dent Assoc. 2001;132: 12S-8S.

50. Pereira LHM, Adebisi IN, Perez A, Wiebel M, Reis I, Duncan R, et al. Salivary markers and risk factor data: a multivariate modeling approach for head and neck squamous cell carcinoma detection. Cancer Biomarkers. 2011;10: 241-9.

51. Rezaei F, Mozaffari HR, Tavasoli J, Zavattaro E, Imani MM, Sadeghi M. Evaluation of Serum and Salivary Interleukin-6 and Interleukin-8 Levels in Oral Squamous Cell Carcinoma Patients: Systematic Review and MetaAnalysis. J Interferon Cytokine Res. 2019:;:jir.2019.0070.

\section{Publisher's Note}

Springer Nature remains neutral with regard to jurisdictional claims in published maps and institutional affiliations.

\section{Ready to submit your research? Choose BMC and benefit from}

- fast, convenient online submission

- thorough peer review by experienced researchers in your field

- rapid publication on acceptance

- support for research data, including large and complex data types

- gold Open Access which fosters wider collaboration and increased citations

- maximum visibility for your research: over $100 \mathrm{M}$ website views per year

At BMC, research is always in progress.

Learn more biomedcentral.com/submissions 